\title{
EFECTO DE PRE-CULTIVOS HOSPEDEROS Y NO HOSPEDEROS EN EL CRECIMIENTO Y PROPÁGULOS MICORRÍCICOS DE TRIGO EN ANDISOL E INCEPTISOL DE CHILE
}

\section{EFFECT OF HOST OR NON-HOST PREVIOUS-CROP IN GROWTH AND MYCORRHIZAL PROPAGULE IN WHEAT IN CHILEAN ANDISOL AND INCEPTISOL SOILS}

\author{
Claudia Castillo ${ }^{1,2^{*}}$, Ángel Montoya ${ }^{1}$, y Fernando Borie ${ }^{3}$ \\ ${ }^{1}$ Escuela de Agronomía, Facultad de Recursos Naturales, Universidad Católica de Temuco, Casilla 15- \\ D, Temuco, Chile. \\ ${ }^{2}$ Núcleo de Investigación en Producción Alimentaria, Universidad Católica de Temuco, Casilla 15-D, \\ Temuco, Chile. \\ ${ }^{3}$ Scientific and Technological Bioresource Nucleus (BIOREN), Casilla 54-D Universidad de La Frontera. \\ * Autor para correspondencia E-mail: ccastill@uct.cl
}

\section{RESUMEN}

Los hongos micorrícicos arbusculares (MA) son simbiontes obligados que favorecen la absorción de nutrientes por la planta. Las rotaciones con cultivos no hospederos pueden afectar la simbiosis. El objetivo del trabajo fue estudiar la influencia de cultivos cabecera de rotación hospederos y no hospederos micorrícicos sobre el número de propágulos fúngicos cultivados en un segundo año con trigo. En el primer año, en invernadero, se sembraron como cultivos cabecera: avena, lupino y raps en un Andisol e Inceptisol de la Región de La Araucanía, utilizando trigo para el segundo año de la rotación. Los tratamientos fueron las rotaciones: avena-trigo (A-T), lupino-trigo (L-T) y rapstrigo (R-T). En el Andisol con L-T, rotación no hospedero-hospedero, se obtuvo el mayor peso aéreo y de inflorescencias de la planta y mayor contenido de P y Fe foliar. En el Inceptisol, la rotación con hospederos (A-T) aumentó significativamente el contenido de $\mathrm{Mg}$ y $\mathrm{K}$. En relación con los parámetros fúngicos, en el Andisol para el tratamiento L-T, se observó la mayor colonización en las raíces del trigo (35\%) mientras que con $\mathrm{A}-\mathrm{T}$ se detectó la mayor densidad de esporas, pero la menor colonización por hongos MA (19\%). La mayor actividad fosfatásica exudada por las raíces del trigo se alcanzó en los dos suelos con R-T, presentando la menor exudación de enzima el Andisol con L-T. Se concluye que la densidad de esporas disminuye en ambos suelos con rotaciones cortas que incluyen cultivos no hospederos, en tanto que aumentó con hospederos continuos.

Palabras clave: avena, lupino, raps, trigo, actividad fosfatásica, Brassicaceae

\section{ABSTRACT}

Arbuscular mycorrhizal (AM) fungi are obligate symbionts that improve nutrient absorption of plants. Including non-mycorrhizal plants in crop rotations as previous-crops can affect the symbiosis. The objective of this work was to study the influence of prior crop with an AM host or a non-host plant on the mycorrhizal propagule number, growth and nutrient uptake in wheat grown in the second year under greenhouse conditions. Oats, lupin and rapeseed were seeded the first year in an Andisol and an Inceptisol of La Araucanía Region, Chile, followed by wheat in the second year. The treatments were: oats-wheat (A-T), lupin-wheat (L-T) and rapeseed-wheat (R-T). The highest above ground biomass, number of inflorescences of plants, and $\mathrm{P}$ and Fe contents in leaves were obtained 
in L-T (cropping with a non-host followed by a host plant) in an Andisol. A significant increase in the content of $\mathrm{Mg}$ and $\mathrm{K}$ in wheat was observed in the A-T treatment in an Inceptisol. Regarding mycorrhizal parameters, the highest root colonization $(35 \%)$ in wheat was observed in L-T in the Andisol, while the A-T treatment recorded the highest spore number but the lowest root colonization $(\mathbf{1 9 \%})$. The highest phosphatase activity of wheat roots was observed in R-T in both types of soils, while the lowest value recorded in L-T in the Andisol. It is was concluded that growing wheat after a non-mycorrhizal host plant, like rapeseed, decreases the number of mycorrhizal propagules (spores) in both Andisol and Inceptisol, while pre-cropping with host plants increases the propagule number in wheat.

Key words: oats, lupin, rapeseed, wheat, phosphatase activity, Brassicaceae.

\section{INTRODUCCIÓN}

En Chile, los suelos derivados de cenizas volcánicas cubren una superficie de más de 5,3 x $10^{6}$ ha incluyendo alrededor del 50 a $60 \%$ de los suelos agrícolas. Entre ellos, uno de los principales órdenes encontrados en el sur del país son los Andisoles, caracterizados por su alta capacidad de fijación de fósforo (P), altos contenidos de materia orgánica $(\mathrm{MO})$ y la mayoría de las veces con niveles elevados de acidez provocando fitotoxicidad por aluminio (Al), manganeso $(\mathrm{Mn})$, y protones $\left(\mathrm{H}^{+}\right)$, junto con deficiencias de algunos nutrientes minerales esenciales como $\mathrm{P}$, calcio $(\mathrm{Ca})$, magnesio $(\mathrm{Mg})$ y molibdeno $(\mathrm{Mo})$. El $\mathrm{Al}$, altamente tóxico especialmente a nivel de la raíz, reduce la captación de agua y nutrientes por la presencia de iones $\mathrm{Al}^{3+}, \mathrm{Al}(\mathrm{OH})^{2+}$ y $\mathrm{Al}(\mathrm{OH})_{2}^{+}$, especies dependientes del $\mathrm{pH}$ (Borie et al., 2010). Otro orden de suelo, son los Inceptisoles, con un perfil un poco más evolucionado que los Entisoles, pero aún con un desarrollo incipiente y con bajo contenido de MO (Tosso, 1985).

En estos suelos resulta relevante la actividad de los hongos micorrícicos arbusculares (MA), simbiontes obligados que habitan las células corticales de las raíces de plantas vasculares y que favorecen la absorción de elementos poco móviles, como P (Smith y Read, 2008), nitrógeno (N) (Bücking y Kafle, 2015) y algunos microelementos (Clark y Zeto, 2000), entre ellos cobre (Cu) (Wang et al., 2005) y zinc (Zn) (Chen et al., 2003). La simbiosis micorrícica natural resulta beneficiosa para la producción de cultivos, especialmente leguminosas, que por el tipo de raíces, absorben menos $\mathrm{P}$, en comparación con cultivos de gramíneas (Isobe et al., 2014).

Un criterio importante para mejorar la producción de los cultivos es la conservación de la densidad de esporas fúngicas en el suelo del agro-ecosistema. Distintas prácticas agronómicas pueden afectar la colonización de las raíces y las esporas de hongos MA, como el sistema de cultivo utilizando ya sea gramíneas o leguminosas, y la rotación con plantas hospederas y no hospederas
(Castillo et al., 2006). Se ha informado que los propágulos fúngicos aumentan después de un cultivo con hospederos micorrícicos (Arihara y Karasawa, 2000). Uchida et al. (2011) informaron que al usar trigo como cabecera de rotación este resultó ser un cultivo eficiente para mantener la densidad de esporas y aumentar las tasas de colonización del cultivo siguiente. Por el contrario, después de un cultivo no hospedero, la tasa de colonización y abundancia de esporas de hongos MA fueron afectadas por la presencia de plantas no micorrícicas (Usuki y Yamamoto, 2003). Según Oyetunji y Osonubi (2007) el efecto que tiene el cultivo hospedero o no hospedero sobre los propágulos fúngicos dependerá del número de años que la planta sea incorporada en la rotación.

Desde una perspectiva de producción agrícola sostenible es importante conocer la forma en que las rotaciones de cultivo afectan la dinámica de los hongos MA, debido a que este manejo, en general, presenta aspectos beneficiosos al mantener o mejorar la fertilidad del suelo disminuyendo la dependencia del cultivo a los agro-químicos. En suelos derivados de cenizas volcánicas del país, existen escasos antecedentes sobre el efecto que tienen las rotaciones de cultivo con plantas hospederas y no hospederas sobre los propágulos de hongos MA. Por otra parte, como el trigo es un cereal muy dependiente de las micorrizas, se podrían obtener bajos rendimientos al establecer una rotación que incluyera como cultivos cabecera por ejemplo, raps o lupino, no hospederos que generan sustancias alelopáticas para el hongo, lo que podría resultar en una rotación poco beneficiosa.

De acuerdo con lo anterior, el objetivo principal de este trabajo fue estudiar, en invernadero, la influencia de cultivos cabecera de rotación que actúan como hospederos micorrícos y no micorrícicos, sobre el número de propágulos fúngicos en un Andisol e Inceptisol de la Región de La Araucanía cultivados en el segundo año con trigo Triticum aestivum L. 


\section{MATERIALES Y MÉTODOS}

El estudio se realizó en los invernaderos y laboratorios de la Escuela de Agronomía, campus San Juan Pablo II de la Universidad Católica de Temuco, y correspondió a un segundo año de rotación de cultivos con plantas hospederas y no hospederas de hongos MA. En el primer año de la rotación se usaron como cultivos cabecera: Avena sativa 'Lord' (cultivo micorrícico), Lupinus albus 'Rumbo' y Brassica napus 'Arthur' (los dos últimos, plantas no hospederas) cultivados en un Andisol serie Temuco y en un Inceptisol serie Lumaco en macetas de $5 \mathrm{~kg}$ de capacidad, mantenidas en condiciones de invernadero.

En nuestro estudio se utilizó trigo (Triticum aestivum L.) ‘Otto-Baer' cultivo dependiente de las micorrizas y abarcó el segundo año de la rotación. Después de la cosecha de los tres cultivos cabecera: avena (A), lupino (L) y raps (R), las macetas conteniendo el Andisol e Inceptisol, permanecieron en barbecho en el exterior del invernadero hasta la siembra del trigo, siendo nuevamente trasladadas al interior del invernadero. Los suelos de cada maceta se removieron y se sembraron ocho semillas pre-germinadas y esterilizadas previamente con $\mathrm{NaOCl} 70 \mathrm{mM}$ por $5 \mathrm{~min}$.

Los tratamientos usados fueron tres: a) trigo después de avena (A-T), b) trigo después de lupino (L-T) y c) trigo después de raps (R-T) en el Andisol (And) e Inceptisol (Inc) usados el primer año de la rotación. Para cada tratamiento se establecieron cinco repeticiones, con un total de 30 unidades experimentales, en un diseño completamente al azar.

Durante todo el tiempo que permaneció el ensayo en el invernadero, la humedad de las macetas se mantuvo a capacidad de campo y bajo condiciones controladas de temperatura (promedio de $23^{\circ} \mathrm{C}$ ) y fotoperiodo de $16 \mathrm{~h}$. La cosecha del trigo se realizó cuando el cultivo alcanzó la madurez fisiológica (etapa 9; Zadoks, 1974).

\section{Parámetros morfoagronómicos}

A la cosecha del trigo, se midió la altura de la planta con un vernier y se contabilizó el número de espigas; luego se separó la parte aérea de la raíz para determinar en estufa (Memmert ${ }^{\circledR}$ D-91126 Schwabach, Alemania) a $70^{\circ} \mathrm{C}$ y $48 \mathrm{~h}$, el peso seco de ambas partes en balanza analítica digital (Denver Instrument Company ${ }^{\circledR}$ AA-200 Colorado, USA).

\section{Parámetros nutricionales}

Macro y micronutrientes en los tejidos de la planta. Una fracción de la parte aérea seca (tallo, espigas, inflorescencias) se molió y se calcinó en mufla (Vulcan ${ }^{\circledR}$ A-550 Yucaipa, USA) a $480^{\circ} \mathrm{C}$ por $8 \mathrm{~h}$. El residuo se trató con una mezcla ácida de ácido nítrico y ácido clorhídrico concentrados evaporando hasta obtención de cenizas blancas. Estas cenizas se disolvieron con la mezcla ácida usada anteriormente, y luego se filtraron. En el filtrado se determinaron los macro $(\mathrm{Ca}, \mathrm{Mg}, \mathrm{K})$ y micronutrientes $(\mathrm{Cu}, \mathrm{Zn}, \mathrm{Fe}, \mathrm{Mn})$ con posterior lectura en espectrofotómetro de absorción atómica (Unicam 969, Pittsburgh, PA, USA) y en el mismo filtrado se determinó $\mathrm{P}$ por espectrofotometría visible (Shimadzu UV-150-02) a $700 \mathrm{~nm}$ (Sadzawka et al., 2004). El contenido mineral se calculó como producto del peso seco aéreo por la concentración foliar del elemento en cada maceta.

\section{Parámetros fúngicos}

Colonización por hongos MA. En una fracción de raíz húmeda se determinó el porcentaje de colonización por hongos MA en las raíces del trigo usando el método del intercepto de líneas (Giovannetti y Mosse, 1980). Se cortaron al azar trozos de raíces de aproximadamente un centímetro de longitud, los que luego se clarificaron con solución de $\mathrm{KOH} 2,5 \%$; posteriormente, se neutralizaron con $\mathrm{HCl} 1 \%$ y finalmente, se tiñeron con azul de tripán al 0,05\% (Phillips y Hayman, 1970). En lupa estereoscópica (Nikon SMZ-2B) a 40x, se observó la presencia $\mathrm{o}$ ausencia de estructuras fúngicas (micelio, esporas o vesículas), calculando el porcentaje de colonización micorrícica.

Número de esporas de hongos MA. De cada maceta se extrajeron las esporas usando $20 \mathrm{~g}$ de suelo rizosférico mediante el método del tamizado húmedo y decantación descrito por Gerdemann y Nicolson (1963). Las esporas de los hongos MA se hicieron pasar a través de una serie de tamices desde 425, 250, 106, 53 y 32 m, y luego se lavaron con abundante agua destilada. Las últimas fracciones de suelo recolectados (tamices de 53 y $32 \mu \mathrm{m}$ ) se transfirieron a tubos plásticos de 50 $\mathrm{mL}$ para inyectarles una solución de sacarosa al $70 \%$ y luego, centrifugarlas (centrífuga Sorvall ${ }^{\circledR}$ T6000 B Waltham, USA) a $2500 \mathrm{rpm}$ durante 7 min (Sieverding, 1991). Finalmente, las esporas se contabilizaron en placa de Doncaster bajo lupa estereoscópica expresando el resultado en número de esporas $100 \mathrm{~mL}^{-1}$ de suelo seco (por medio de la determinación de la densidad aparente del suelo de cada maceta).

\section{Parámetros edáficos}

pH. Se midió potenciométricamente (Microprocessor $\mathrm{pH}$ meters HANNA Instruments ${ }^{\circledR} 211$ Texas, USA) en una suspensión suelo:agua en relación 1:2,5 (Zagal y Sadzawka, 2007). 

en trigo en Andisol e Inceptisol de Chile

P disponible. El $\mathrm{P}$ se analizó mediante extracción con $\mathrm{NaHCO}_{3} 0,5 \mathrm{M}$ a pH 8,5 y posterior lectura espectrofotométrica a $700 \mathrm{~nm}$ (Olsen y Sommers, 1982).

\section{Parámetros bioquímicos}

Actividad fosfatásica ácida. La actividad fosfatásica ácida en los suelos se determinó usando $p$-nitrofenilfosfato de acuerdo al procedimiento descrito por Tabatabai y Bremner (1969), con modificaciones para suelos volcánicos con altos contenidos de $\mathrm{MO}$ informadas por Rubio et al. (1990) cuantificándose la cantidad de $p$-nitrofenol liberado espectrofotométricamente a $400 \mathrm{~nm}$.

\section{Análisis estadístico}

Los resultados obtenidos de cada variable investigada se analizaron a través de un análisis de varianza ANDEVA de una vía y posterior test de Tukey de rango múltiple $(\mathrm{p} \leq 0,05)$.

\section{RESULTADOS Y DISCUSIÓN}

En ambos suelos (And e Inc), no se encontraron diferencias en el crecimiento del trigo, pero en Inc, las plantas alcanzaron alturas superiores a los 45 cm en los tratamientos A-T y R-T (Fig. 1A). En And, para L-T y R-T, el peso seco aéreo de las plantas fue mayor que en Inc presentando L-T diferencias en masa que alcanzaron 35\% sobre A-T (Fig. 1B). Estos resultados coinciden con lo informado por Angus et al. (2008) quienes compararon el efecto sobre el rendimiento del trigo de dos precultivos hospederos de hongos MA (lino, Linum usitatissimum y haba, Vicia faba) con dos precultivos no hospederos (raps y lupino) crecidos en rotación y encontraron que el rendimiento del trigo después de los no hospederos fue mayor que después de los hospederos micorrícicos.

Probablemente, en el And que presentó inicialmente un mayor contenido de $\mathrm{P}$ total que el Inc, en la rotación A-T, en el cultivo hospedero avena, las hifas micorrícicas aumentaron el volumen explorado por las raíces, mientras que en la rotación L-T la raíz pivotante del lupino exploró comparativamente un volumen menor de suelo. Sin embargo, las raicillas densamente empaquetadas de esta leguminosa pudieron unirse estrechamente a las partículas de MO movilizando $\mathrm{P}$ inorgánico desde la fracción escasamente soluble, mediante excreción de ácidos orgánicos y fosfatasas ácidas. Lo anterior podría indicar que las plantas de trigo percibieron esta diferencia en la química del ambiente de la rizósfera provenientes de los cultivos cabecera. En el caso de lupino, el desarrollo de las raíces proteoídeas aumentó posiblemente el P disponible para el cultivo de trigo que lo siguió en la rotación, y como consecuencia aumentó la biomasa.

Por el contrario, la masa radical fue mayor en Inc que en And, pero sin diferencias entre tratamientos (Fig. 1B). Además, en And, se contabilizó un mayor número de espigas en el tratamiento $\mathrm{A}-\mathrm{T}$, con 10 espigas, mientras en los otros tratamientos se encontraron 9 y en Inc, los tres tratamientos, tuvieron 8 espigas. En And, el peso de las inflorescencias fue mayor con diferencias significativas entre L-T y R-T, ambos tratamientos provenientes de cultivos no micorrizables. En Inc, la mayor masa de inflorescencias se obtuvo con A-T, presentando diferencias con los otros tratamientos (Fig. 1C). En la relación raíz/tallo (R/S), sólo se encontraron diferencias en And, entre A-T, que tuvo la mayor relación, con L-T y R-T. En Inc, la relación fue mayor, señalando un mayor crecimiento radical, que en And (Fig. 1D). Cuando el $\mathrm{P}$ es deficiente, como en Inc, las plántulas tienen un volumen de raíces relativamente grande y presentan una mayor relación R/S. Los hongos MA, al mejorar la captación de nutrientes favorecen el incremento de biomasa vegetal influyendo en la relación de distribución R/S, que es menor, cuando más efectiva es la simbiosis como ocurrió en And con L-T y R-T. Probablemente, también en la menor relación R/S en And, influyeron las diferencias físico-químicas que existen entre los dos suelos (Tabla 1).

Por otra parte, el contenido de nutrientes en la planta refleja la movilidad de los elementos desde el suelo hacia la fitomasa de los cultivos (Fig. 2 y Fig. 3). En And, (Fig. 2A) el P en L-T, fue significativamente mayor que en los otros tratamientos, mientras el contenido de $\mathrm{Mg}$, en $\mathrm{L}-\mathrm{T}$, sólo presentó diferencias con A-T (Fig. 2C). Por el contrario, el contenido de $\mathrm{K}$ fue estadísticamente significativo en A-T en relación a L-T (Fig. 2D). En Inc, los contenidos de macronutrientes fueron menores que en And, excepto para $\mathrm{K}$, que fue significativamente mayor en A-T (Fig. 2D). El otro nutriente que presentó diferencias fue $\mathrm{Mg}$, en Inc, con mayor contenido en A-T (Fig. 2C).

En ambos suelos, en la rotación A-T, la relación inversa que se observó entre el número de esporas y el contenido de $\mathrm{K}$ podría sugerir una modificación más biológica que química a nivel de la rizósfera. La disponibilidad de nutrientes y cambios cuantitativos o cualitativos en los exudados de las raíces pueden afectar a las poblaciones microbianas que presentan distintas necesidades de nutrientes.

En relación a los contenidos de micronutrientes, And presentó diferencias en $\mathrm{R}-\mathrm{T}$, con $\mathrm{Cu}$ significativamente mayor que A-T (Fig. 3A) y Fe, en L-T, mayor que R-T (Fig. 3C). Al comparar los suelos iniciales (Tabla 1), Inc presentó un elevado 
Tabla 1. Características físicas y químicas de los suelos Andisol e Inceptisol al comienzo de la rotación de cultivos*.

Table 1. Physico-chemicals characteristics of the Andisol and Inceptisol at the beginning of crop rotation*.

\begin{tabular}{|c|c|c|c|c|c|}
\hline Tipo de suelo & $\mathrm{pH}$ & Dap & P total & $\mathrm{Fe}$ & Al \\
\hline & & $\mathrm{g} \mathrm{mL}^{-1}$ & --------- & $\mathrm{mg} \mathrm{kg}^{-1}$ & ---------- \\
\hline Andisol & 5,79 & 0,40 & 3182 & 12,71 & 16,10 \\
\hline Inceptisol & 5,05 & 0,98 & 1008 & 19,47 & 64,05 \\
\hline
\end{tabular}

${ }^{*}$ Castillo et al. (2008).
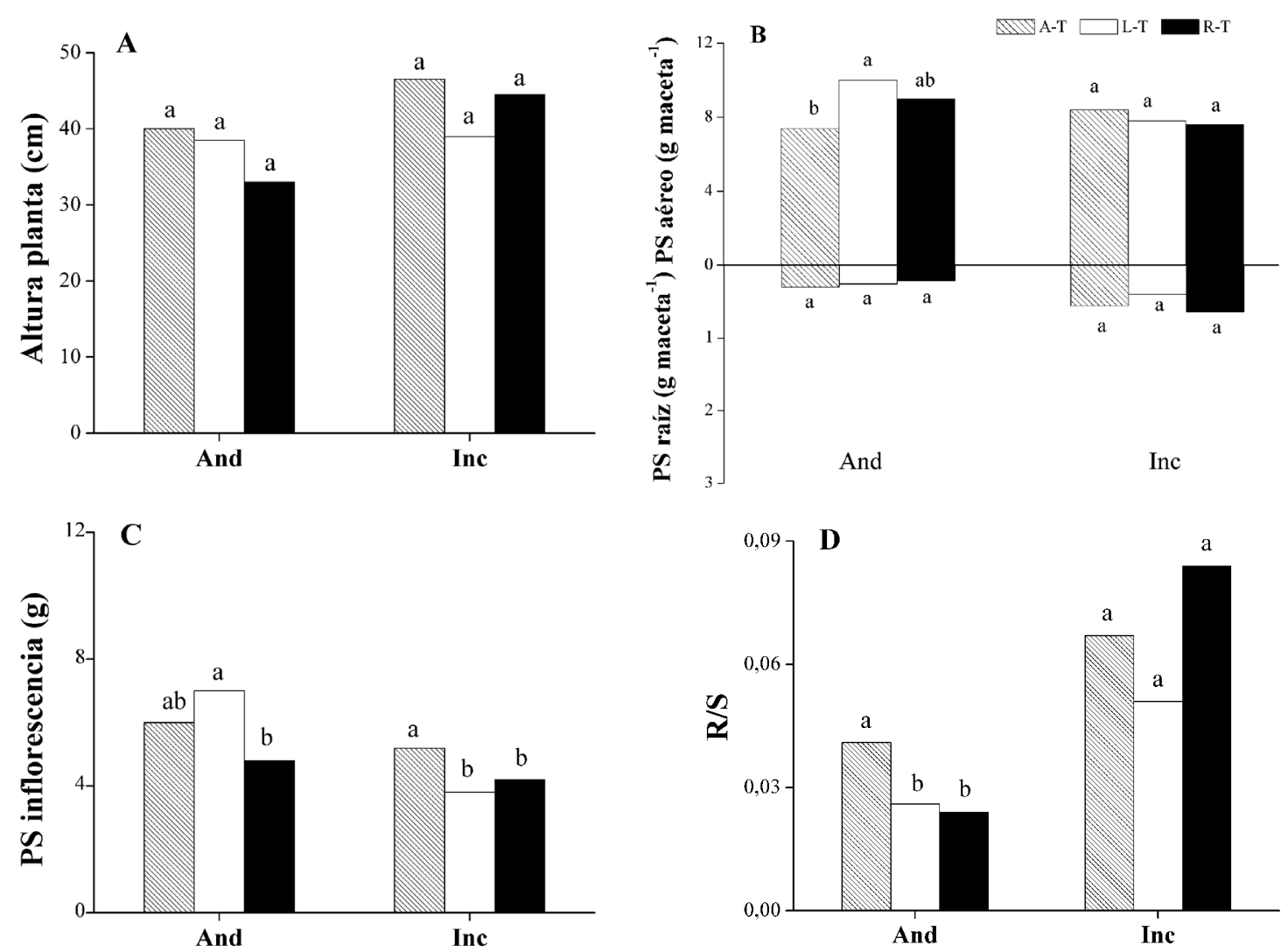

Fig. 1. Parámetros morfoagronómicos en trigo. A) altura de planta; B) peso aéreo y peso raíz; C) peso inflorescencias; y D) relación raíz/tallo en un Andisol (And) e Inceptisol (Inc), al segundo año de una rotación usando como pre-cultivos avena (A-T), lupino (L-T) y raps (R-T).

Fig. 1. Morphoagronomic parameters in wheat: A) plant height, B) shoot and root dry weight, inflorescence weight, and root-shoot relationship in an Andisol (And) and Inceptisol (Inc), in the second year of a crop rotation system using oats, lupin and rapeseed as pre-crops.

Para cada suelo, columnas seguidas de distinta letra presentan diferencias significativas según Test de Tukey $(\mathrm{p} \leq 0,05)$. 

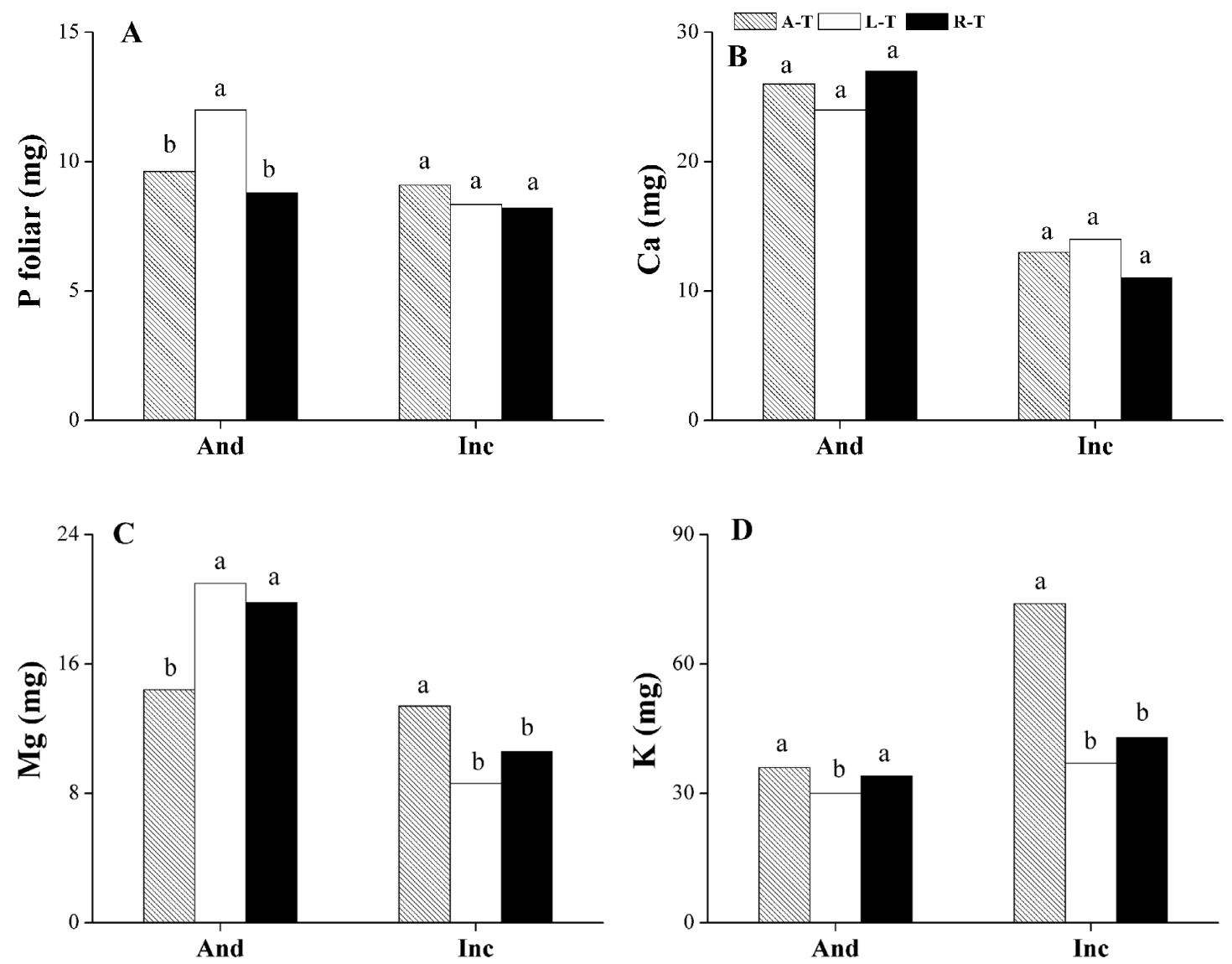

Fig. 2. Contenido de macronutrientes foliares en plantas de trigo: A) $\mathrm{P}$; B) $\mathrm{Ca}$; C) $\mathrm{Mg}$; y D) $\mathrm{K}$, en un Andisol (And) e Inceptisol (Inc), al segundo año de una rotación usando como pre-cultivos avena (A-T), lupino (L-T) y raps (R-T).

Fig. 2. Macronutrient content in leaves of wheat plants: A) $P$, B) Ca, C) $\mathrm{Mg}$, and D) $K$, in an Andisol (And) and Inceptisol (Inc), in the second year of a crop rotation system using oats (A-T), lupin (L-T) and rapeseed (R-T) as previous crops.

Para cada suelo, columnas seguidas de distinta letra presentan diferencias significativas según Test de Tukey $(\mathrm{p} \leq 0,05)$.

$\mathrm{Al}$, siendo $\mathrm{Al}$ y $\mathrm{Mn}$, elementos fitotóxicos y muy abundantes en suelos ácidos, principalmente en Andisoles del sur de Chile (Borie et al., 2002). En la Fig. 3D, se puede observar que en And, la disminución en el contenido de Mn podría atribuirse a una serie de mecanismos simbióticos basados en el enlazamiento del metal al micelio extraradical fúngico desarrollado alrededor de las raíces (Janousková et al., 2006).

Guadarrama et al. (2004) informaron que los hongos MA aumentan la capacidad de adquisición y asimilación de recursos por parte de la planta hospedera, debido a que las hifas externas de los hongos, en comparación con las raíces, poseen mayor habilidad para explorar el suelo, mejorando la captación de nutrientes. Según Clark y Zeto (2000) la adquisición de $\mathrm{K}, \mathrm{Ca}, \mathrm{Mg}$, Zn y Cu se puede incrementar por inoculación con hongos micorrícicos en suelos ácidos.

En general, los resultados del presente estudio respecto a parámetros morfoagronómicos y nutricionales no presentaron grandes diferencias entre los tres tratamientos al incluir plantas hospederas y no hospederas de micorrizas. De acuerdo con antecedentes de la literatura, en muchos ensayos en maceta, se observó que las plantas absorben $P$ para sus propios requerimientos y después de la madurez dejan $\mathrm{P}$ disponible en el suelo, el que puede ser absorbido por el cultivo siguiente. Según Hocking (2001), por este tipo de mecanismo, el lupino aumentó la absorción de $\mathrm{P}$ y el crecimiento del cultivo siguiente que fue trigo. Esta capacidad conocida como equilibrio funcional es una reacción común de las plantas, 

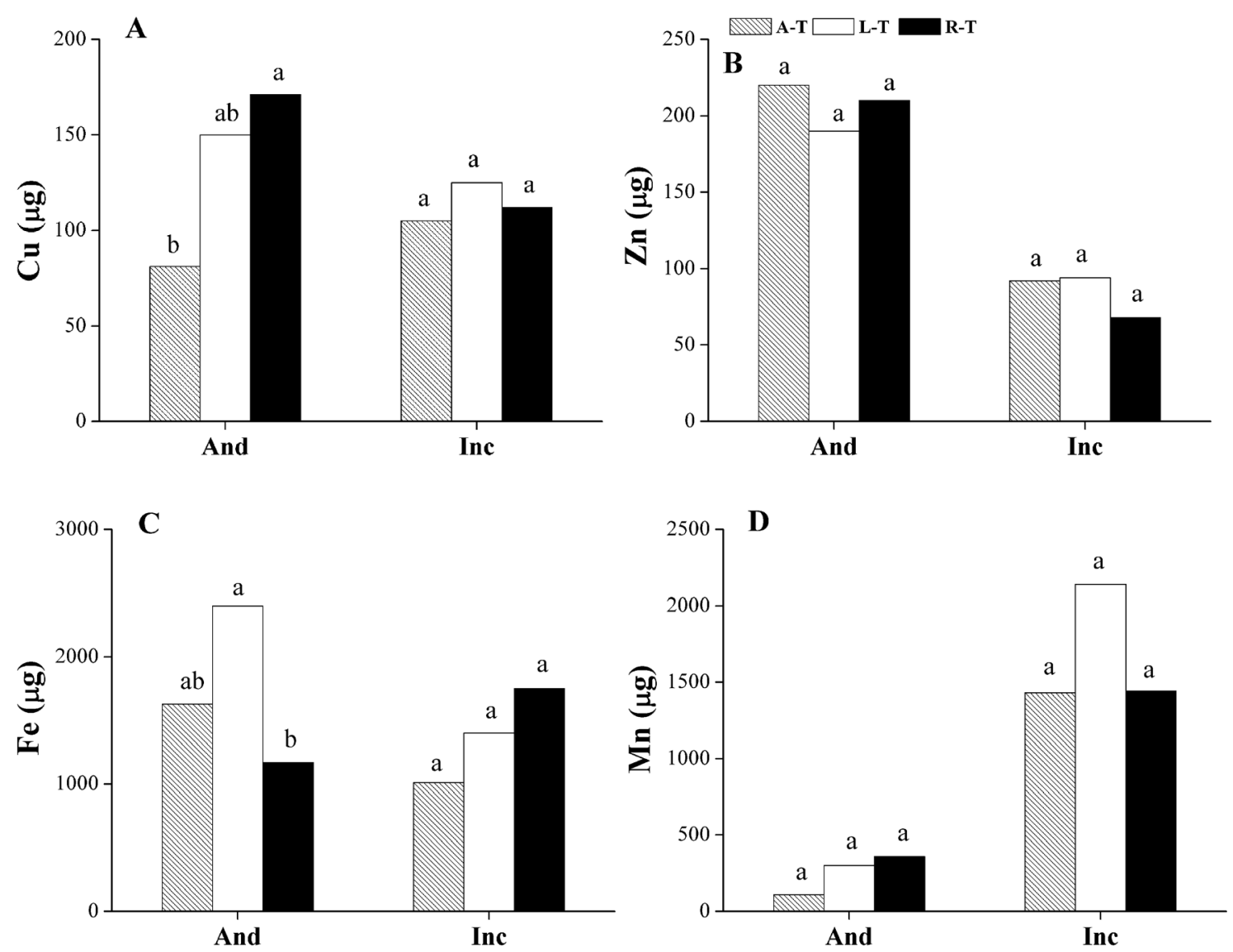

Fig. 3. Contenido de micronutrientes foliares en plantas de trigo: A) $\mathrm{Cu}$; B) $\mathrm{Zn}$; C) Fe; y D) Mn, en un Andisol (And) e Inceptisol (Inc), al segundo año de una rotación usando como pre-cultivos avena (A-T), lupino (L-T) y raps (R-T).

Fig. 3. Micronutrient content in leaves of wheat plant: $\mathrm{Cu}, \mathrm{A}), \mathrm{Zn}, \mathrm{B}), \mathrm{Fe}, \mathrm{C})$, and $\mathrm{Mn} \mathrm{D}$ ), in an Andisol (And) and Inceptisol (Inc), in the second year of a crop rotation system using oats (A-T), lupin $(\mathrm{L}-\mathrm{T})$, and rapeseed $(\mathrm{R}-\mathrm{T})$ as previous crops.

Para cada suelo, columnas seguidas de distinta letra presentan diferencias significativas según Test de Tukey $(p \leq 0,05)$.

que les permite asignar el crecimiento de forma selectiva a los órganos que captan más de un recurso limitante (Angus et al., 2015).

En el primer año de la rotación, la colonización por hongos MA en las raíces de avena, en And alcanzó 35\%, mientras que en Inc fue levemente menor, con 31\% (Castillo et al., 2008). En este estudio que abarcó el segundo año de la rotación, en And, la mayor colonización en las raíces del trigo correspondió al tratamiento L-T, presentando diferencias significativas con A-T que sólo alcanzó un $19 \%$ (Fig. 4A). En Inc, no se encontraron diferencias entre los tres tratamientos siendo los porcentajes más bajos que en And, para las rotaciones provenientes de cultivos no hospederos (Fig. 4A). Los resultados en Inc, concuerdan con lo informado por Ryan et al. (2005) quienes en trigo encontraron niveles similares de colonización siguiendo cultivos hospederos o no hospederos. En And, la mayor colonización en L-T resultó contraria a lo informado en la literatura, que cuando trigo sigue al no hospedero, la colonización es baja. En regiones templadas de Australia, trigo seguido de Brasicáceas presentó menor colonización que trigo seguido de trigo, o de otros cultivos hospederos (Ryan y Graham, 2002; Ryan y Angus, 2003; Ryan y Kirkegaard, 2012).

La mayor colonización HMA alcanzada en la rotación L-T benefició la biomasa del trigo; probablemente debido a que las hifas del micelio externo tuvieron la capacidad de captar y transportar a las raíces del cereal el $\mathrm{N}$ que permaneció en el suelo, proveniente de la 
nodulación del Rhizobium en simbiosis con la leguminosa.

En ambos suelos, en relación al otro parámetro fúngico, las esporas del hongo MA, se observó una disminución de propágulos cuando se incluyó como cultivo cabecera una planta no hospedera, especialmente el raps (Fig. 4B). La cantidad de esporas en el tratamiento L-T varió de acuerdo con el tipo de suelo; And no presentó diferencias con A-T, pero en Inc se encontraron diferencias significativas entre los dos tratamientos (Fig. 4B). En general, al comparar los tratamientos en And con los respectivos tratamientos en Inc, la cantidad de esporas fue mayor en el Andisol. En And, la cantidad de propágulos en A-T en relación con R-T, alcanzaron diferencias de 300\%. En Inc no se encontraron diferencias en los tratamientos con no hospederos, pero sí hubo diferencia entre estos últimos con $\mathrm{A}-\mathrm{T}$, aumentando los propágulos fúngicos en aproximadamente $240 \%$ en la rotación con hospederos. En Inc la baja cantidad de esporas detectadas en L-T y R-T $y$ en And en R-T, coinciden con antecedentes de la literatura sobre la densidad de esporas de hongos MA, que generalmente es menor en suelos sembrados con cultivos no hospederos, que en suelos cultivados con plantas hospederas de hongos MA. Una rotación de cultivos corta, que incluye no hospederos, puede alterar el potencial inherente de hongos MA en el suelo, influyendo significativamente en la simbiosis del cultivo posterior, por cambio en la masa de raíces micorrizadas y número de esporas (Panja y Chaudhuri, 2004). En Inc la menor densidad de esporas fúngicas en el tratamiento R-T, concuerda con estudios que han informado que con cultivos de Brasicáceas se reduce la colonización de raíces y el número de esporas del cultivo siguiente (Harinikumar y Bagyaraj, 1988; Ryan y Graham, 2002). Lo anterior podría explicarse porque esta familia, que es no hospedera de hongos MA y a la cual pertenece el raps, contienen en sus raíces glucosinolatos, que al momento de la preparación del suelo o después de la cosecha liberan isotiocianatos. Estos compuestos poseen actividad biocida que incluye efectos fitotóxicos (Petersen et al., 2001), nematicidas (Henderson et al., 2009) y fungicidas (Dunne et al., 2003). Algunas investigaciones han informado que el sistema de cultivo y la rotación afectan las esporas y colonización de las raíces (Jeffries y Barea, 2001); pero, al incluir plantas hospederas en la rotación se pueden mantener las comunidades fúngicas con incremento en el rendimiento del cultivo siguiente (Vestberg et al., 2005).

Además de la simbiosis micorrícica, las plantas utilizan otras estrategias para la captación de nutrientes, especialmente $\mathrm{P}$, como exudación de enzimas desde las raíces, entre ellas, las fosfatasas, que catalizan la hidrólisis de ésteres y anhídridos del ácido fosfórico, junto con mineralización de $\mathrm{P}$ orgánico y liberación de P disponible. En general (Fig. 5) se puede observar que la actividad fosfatásica ácida fue mayor en Inc (con bajo contenido de $\mathrm{P}$ ), que en And y entre ambos suelos la mayor liberación de enzima se cuantificó en
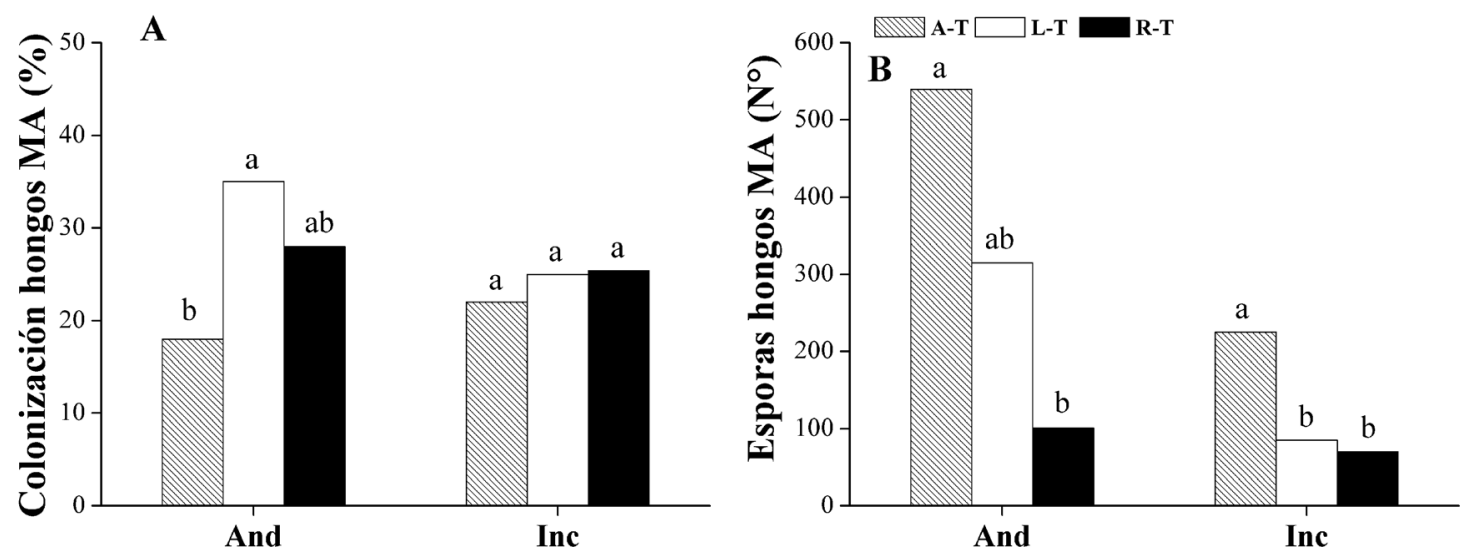

Fig. 4. A) Colonización por hongos micorrícicos arbusculares (MA) en raíces de plantas de trigo; y B) número de esporas en un Andisol (And) e Inceptisol (Inc), al segundo año de una rotación usando como pre-cultivos avena (A-T), lupino (L-T) y raps (R-T).

Fig. 4. A) Arbuscular mycorrhizal fungi colonization in roots of wheat plants; and B) spore number in an Andisol (And) and Inceptisol (Inc), in the second year of a crop rotation system using oats (A-T), lupin (L-T), and rapeseed (R-T) as previous crops.

Para cada suelo, columnas seguidas de distinta letra presentan diferencias significativas según Test de Tukey $(p \leq 0,05)$. 


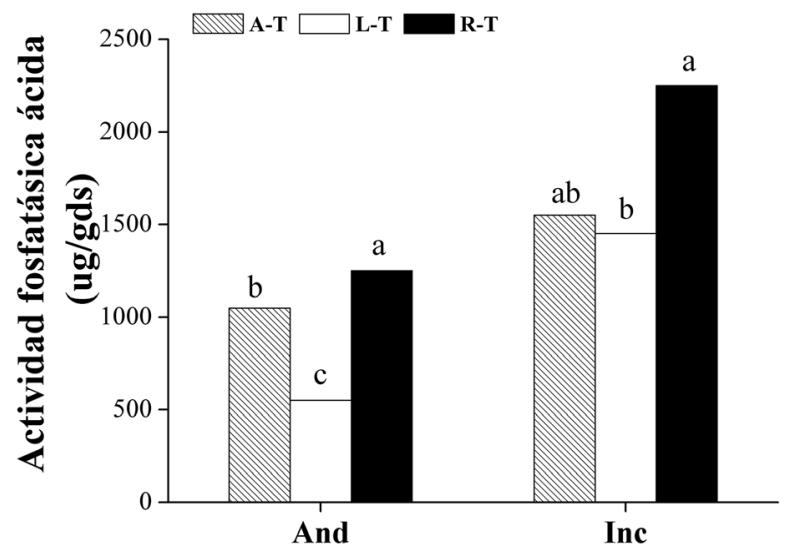

Fig. 5. Actividad fosfatásica ácida en un Andisol (And) e Inceptisol (Inc), al segundo año de una rotación usando como pre-cultivos avena (A-T), lupino (L-T) y raps (R-T).

Fig. 5. Acid phosphatase activity in an Andisol (And) and Inceptisol (Inc), in the second year of a crop rotation system using oats (A-T), lupin (L-T), and rapeseed (R-T) as previous crops.

Para cada suelo, columnas seguidas de distinta letra presentan diferencias significativas según Test de Tukey $(\mathrm{p} \leq 0,05)$.

el tratamiento R-T. En And hubo diferencias significativas entre los tres tratamientos siendo $\mathrm{R}-\mathrm{T}>\mathrm{A}-\mathrm{T}>\mathrm{L}-\mathrm{T}$. En Inc la cantidad de enzima exudada en $\mathrm{R}-\mathrm{T}$ fue significativamente mayor que en L-T.

En And con L-T, se produjo un aumento significativo de la colonización fúngica (Fig. 4A), acompañado de un descenso drástico en la actividad fosfatásica (Fig. 5). Por el contrario, en Inc un aumento enzimático (Fig. 5) produjo menor densidad de esporas (Fig. 4B), corroborando que ambos mecanismos actuarían sinérgicamente según lo informado por Rubio et al. (1990).

\section{CONCLUSIONES}

El presente estudio permitió concluir que tanto el pre-cultivo como el tipo de suelo afectaron la densidad de hongos MA. En ambos suelos, las rotaciones con hospederos continuos presentaron la más alta densidad de propágulos, disminuyendo cuando el pre-cultivo fue raps (no hospedero). En el Andisol, la mayor colonización MA en las raíces del trigo se obtuvo en rotaciones con cultivos no hospederos, alcanzando mayores contenidos de $\mathrm{P}, \mathrm{Mg}, \mathrm{Cu}$, Fe y de $\mathrm{Mg}$ y $\mathrm{K}$ en el Inceptisol con hospederos continuos. Este mismo suelo presentó en todas las rotaciones una mayor actividad fosfatásica; sin embargo, en ambos suelos el trigo tuvo una menor actividad enzimática cuando siguió a lupino (no hospedero).

\section{LITERATURA CITADA}

Angus, J.F., J.A. Kirkegaard, J.R. Hunt, M.H. Ryan, L. Ohlander, and M.B. Peoples. 2015. Break crops and rotations for wheat. Crop Pasture Science 66(6):523-552.

Angus, J.F., M.B. Peoples, J.A. Kirkegaard, M.H. Ryan, and L. Ohlander. 2008. The value of break crops for wheat. In Proceedings 14th Australian Agronomy Conference. Australian Society of Agronomy. The Regional Institute, Gosford NSW, Australia.

Arihara, J., and T. Karasawa. 2000. Effect of previous crops on arbuscular mycorrhizal formation and growth of succeeding maize. Soil Science and Plant Nutrition 46:43-51.

Borie, F., Y. Redel, R. Rubio, J.L. Rouanet, and J.M. Barea. 2002. Interactions between crop residues applications and mycorrhizal developments and some soil-root interface properties and mineral acquisition by plants in an acidic soil. Biology and Fertility Soils 36(2):151-160.

Borie, F., R. Rubio, A. Morales, G. Curaqueo, and P. Cornejo P. 2010. Arbuscular mycorrhizae in agricultural and forest ecosystems in Chile. Journal Soil Science and Plant Nutrition 10(3):185-206.

Bücking, H., and A. Kafle. 2015. Role of arbuscular mycorrhizal fungi in the nitrogen uptake of plants: current knowledge and research gaps. Agronomy 5:587-612. 
Castillo, C.G., I. Astroza, F. Borie, y R. Rubio. 2008. Efecto de cultivos hospederos y no hospederos sobre propágulos micorrícicos arbusculares. Journal Soil Science and Plant Nutrition 8(1):37-54.

Castillo, C.G., R. Rubio, J.L. Rouannet, and F. Borie. 2006. Early effects of tillage and crop rotation on arbuscular mycorrhizal fungal propagules in an Ultisol. Biology and Fertility Soils 43:83-92.

Chen, B.D., X.L. Li, H.Q. Tao, P. Christie, and M.H. Wong. 2003. The role of arbuscular mycorrhiza in zinc uptake by red clover growing in calcareous soil spiked with various quantities of zinc. Chemosphere 50(6):839-846.

Clark, R.B., and S.K. Zeto. 2000. Mineral acquisition by arbuscular mycorrhizal plants. Journal Plant Nutrition 23:867-902.

Dunne, C.P., B. Dell, G.E. StJ. Hardy. 2003. The effect of biofumigants on the vegetative growth of five Phytophthora species in vitro. Acta Horticulturae 602:45-51.

Gerdemann, J.W., and T.H. Nicolson. 1963. Spores of mycorrhizal Endogone species extracted from soil by wet sieving and decanting. Transactions of the British Mycological Society 46:235-244.

Giovannetti, M., and B. Mosse. 1980. An evaluation of techniques for measuring vesicular-arbuscular mycorrhizal infection in roots. New Phytology 84:489-500.

Guadarrama, P., I. Sánchez-Gallén, J. ÁlvarezSánchez, y J. Ramos-Zapata. 2004. Hongos y plantas, beneficios a diferentes escalas en micorrizas arbusculares. Ciencias 73:38-45.

Harinikumar, K.M., and D.J. Bagyaraj. 1988. Effect of crop rotation on native vesiculararbuscular mycorrhizal propagules in soil. Plant and Soil 110:77-80.

Henderson, D.R., E. Riga, R.A. Ramírez, J. Wilson, and W.E. Snyder. 2009. Mustard biofumigation disrupts biological control by Steinernema spp. nematodes in the soil. Biology Control 48:316-322.

Hocking, P.J. 2001. Organic acid exuded from roots in phosphorus uptake and aluminium tolerance of plants in acid soils. Advances Agronomy 74:63-97.

Isobe, K., M. Higo, T. Kondo, N. Sato, S. Takeyama, and Y. Torigoe. 2014. Effect of winter crop species on arbuscular mycorrhizal fungal colonization and subsequent soybean yields. Plant Production Science 17(3):260-267.

Janousková, M., D. Pavlíková, and M. Vosátka M. 2006. Potential contribution of arbuscular mycorrhiza to cadmium immobilisation in soil. Chemosphere 65:1959-1965.
Jeffries, P., and J.M. Barea. 2001. Arbuscular mycorrhiza. A key component of sustainable plant-soil ecosystems. p. 95-113. In B. Hock (ed.). The Mycota. IX Fungal Associations. Springer-Verlag, Berlin, Germany.

Olsen, S.R., and L.E. Sommers. 1982. Phosphorus. p. 403-430. In A.L. Page. Methods of Soil Analysis. Part. 2. $2^{\text {nd }}$ ed. Agron. Monogr. 9. ASA and SSSA, Madison, Wisconsin, USA.

Oyetunji, O.J., and O. Osonubi. 2007. Assessment of influence of alley cropping systems and arbuscular mycorrizal (AM) fungi on cassava productivity in derived savanna zone in Nigeria. World Journal of Agricultural Science 3(4):489-497.

Panja, B.N., and S. Chaudhuri. 2004. Exploitation of soil arbuscular mycorrhizal potential for AM dependent mandarin orange plants by pre-cropping with mycotrophic crops. Applied Soil Ecology 26:249-255.

Petersen, J., R. Belz, F. Walker, and K. Hurle. 2001. Weed suppression by release of isothiocyanates from turnip-rape mulch. Agronomy Journal 93:37-43.

Phillips, J.M., and D.S. Hayman. 1970. Improved procedures for clearing roots and staining parasitic and vesicular arbuscular mycorrhizal fungi for rapid assessment of infection. Transactions of the British Mycological Society 55:158-161.

Rubio, R., E. Moraga, and F. Borie. 1990. Acid phosphatase activity and vesicular-arbuscular infection associated with roots of four wheat cultivars. Journal Plant Nutrition 13:585-598.

Ryan, M.H., and J.F. Angus. 2003. Arbuscular mycorrhizae in wheat and field pea crops on a low P soil increased Zn-uptake but no increase in P-uptake or yield. Plant and Soil 250:225-239.

Ryan, M.H., and J.H. Graham. 2002. Is there a role for arbuscular mycorrhizal fungi in production agriculture? Plant and Soil 244:263-271.

Ryan, M.H., and J.A. Kirkegaard. 2012. The agronomic relevance of arbuscular mycorrhizas in the fertility of Australian extensive cropping systems. Agriculture Ecosystems \& Environment 163:37-53.

Ryan, M.H., A.F. van Herwaarden, J.F. Angus, and J.A. Kirkegaard. 2005. Reduced growth of autumn-sown wheat in a low-P soil is associated with high colonisation by arbuscular mycorrhizal fungi. Plant and Soil 270:275-286. 
Sadzawka, A., M. Carrasco, R. Grez, and M.L. Mora. 2004. Métodos de análisis de tejidos vegetales. 53 p. Comisión de Normalización y Acreditación. Sociedad Chilena de la Ciencia del Suelo. INIA La Platina, Santiago, Chile.

Sieverding, E. 1991. Vesicular arbuscular mycorrhiza management in tropical agrosystems. 371 p. Deutsche Gesellschaft für Technische Zusammenarbeiit (GTZ), $\mathrm{GmbH}$, Eschborn, Germany.

Smith, S.E., and D.J. Read 2008. Mycorrhizal symbiosis. 3rd ed. Academic Press, New York, USA.

Tabatabai, M.A., and J.M. Bremner. 1969. Use of $p$-nitrophenyl phosphate for assay of soil phosphatase activity. Soil Biology and Biochemistry 1:301-307.

Tosso, J. 1985. Suelos volcánicos de Chile. 723 p. Instituto de Investigaciones Agropecuarias, Santiago, Chile.

Uchida, T., H. Kobayashi, and N. Yoshino. 2011. Effects of arbuscular mycorrhizal colonization on soybean nutrient uptake during ripening period with barley cover cropping. Japanese Journal of Crop Science 80:277-283.
Usuki, K., and H. Yamamoto. 2003. Effect of cropping system on arbuscular mycorrhizal fungal population, growth and yield of succeeding crop on andosol in central region of Japan. Japanese Journal of Crop Science 72:158-162.

Vestberg, M., K. Saari, S. Kukkonen, and T. Hurme. 2005. Mycotrophy of crops in rotation and soil amendment with peat influence the abundance and effectiveness of indigenous arbuscular mycorrhizal fungi in field soil. Mycorrhiza 15:447-458.

Wang, F.Y., X.G. Lin, and R. Yin. 2005. Influence of arbuscular mycorrhizal fungi on growth and $\mathrm{Cu}$ uptake of Elsholtzia splendens. Huan Jing Ke Xue 26:174-180. Chinese.

Zadoks, J.C. 1974. A decimal code for the growth stage of cereals. Weed Research 14:415-421.

Zagal, E., and A. Sadzawka. 2007. Protocolo de métodos de análisis para suelos y lodos. Universidad de Concepción, Facultad de Agronomía, Chillán, Chile. 